\title{
Method for the diagnosis of high-voltage dielectric elements during operation based on dynamic registration of electromagnetic radiation
}

\author{
Dmitry Ivanov ${ }^{*}$, Tatyana Galieva ${ }^{1}$, Marat Sadykov $^{1}$, Alexander Golenischev-Kutuzov and Anatoly Naumov ${ }^{1}$ \\ ${ }^{1}$ Kazan State Power Engineering University, Russia
}

\begin{abstract}
Complex remote monitoring and the developed non-contact diagnostic device (NDD) allow monitoring the technical condition and revealing the residual life of high-voltage dielectric elements. It is based on non-contact remote periodic measurement of a set of diagnostic parameters (characteristics of electrical discharges emitted by insulators during operation) necessary for a reliable assessment of the operating state of high-voltage dielectric equipment at the moment of operation and determination of its residual life. NDDs are installed on each phase conductor and used to measure diagnostic parameters, followed by computer processing of the accumulated information signals. An analysis of the set of necessary diagnostic parameters makes it possible to form a conclusion on the degree of performance of dielectric elements. The paper considers a method for detecting defective high-voltage insulators using the developed non-contact diagnostic devices by the radio frequency method and assessing the difference in time of arrival of a partial discharge signal.
\end{abstract}

\section{Introduction}

The main goal of electric power companies is to maximize the use of available equipment and minimize huge capital investments. Requirements for reliability and performance are increasing every year. A large percentage of obsolete and worn-out equipment creates serious problems for energy companies. Aging of equipment increases the likelihood of failure, and measures must be taken to remain its operational.

A high proportion of overhead power transmission line failures is caused by damage to insulators. A significant proportion of insulators are approaching or exceeding the service life for which they were designed. Thus, the maintenance and life extension of these aging elements is of particular interest to electric power companies. Because insulators play such a critical role in the reliability of a power system, businesses are increasingly opting for reliable, economical, fast and safe methods for detecting faulty insulators for personnel.

The occurrence of a partial discharge (PD) is a key indicator of defects in the insulation system, since a part of the dielectric gap is shunted during the discharge. The magnitude of the PD intensity, their number and place of occurrence make it possible to judge the residual life of the high-voltage insulator.

As a result of PD, some physical, chemical and acoustic phenomena occurs, and many methods of PD detection are based on their quantitative assessment. One of the consequences of partial discharges is the propagation of radio frequency (RF) radiation of the ultrahigh frequency (UHF) range.

\section{PD localization}

The determination of PDs using radio frequency methods in high voltage equipment has been extensively studied [1-7]. Research in this area has been carried out in cables [8-11], gas and air insulated switchgears [10-11] and transformers $[2,3,12]$, but the use of such methods for external insulators is very limited, while internal localization of $\mathrm{PD}$ requires reliable computation algorithms [1].

To measure microwave radiation, receiving antennas are used, arranged in various forms, then, using mathematical methods, the location of the partial discharge event is triangulated in 3 dimensions.

To detect PD in high-voltage insulators (HVI), it is proposed to use non-contact diagnostic devices (NDD), the block diagram of which is shown in Figure 1. The NDD conducts basic measurements using an electromagnetic (EM) sensor and a phase sensor. The NDD is installed on the phase wire at a predetermined distance from the wire suspension point. The EM sensor uses an antenna to measure the electromagnetic field emitted by the PD.

\footnotetext{
* Corresponding author: divanale@gmail.com
} 


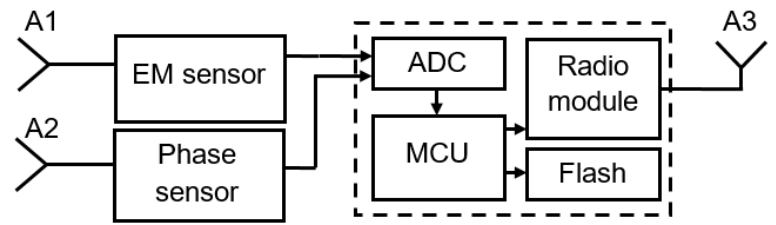

Fig. 1. Block diagram of a non-contact diagnostic device: A1 EM sensor antenna, A2 - phase sensor antenna, A3 - antenna for data transmission, ADC - analog-to-digital converter, $\mathrm{MCU}$ - microcontroller.

Capable of registering high-frequency discharges the EM sensor is built on a RTL-SDR module with a UART interface. Frequencies from 100 to $2000 \mathrm{MHz}$ are considered for the measurement of high-frequency PD pulses. After digital processing in the ADC, the data obtained from the two sensors is processed in the microcontroller and transferred to the storage device (Flash).

The signal is received by the receiving antenna A1 (Fig. 2) and enters the input of the EM sensor after detection. The EM sensor contains an op-amp preamplifier and a comparator. In fig. 2 shows the signal in red, which is received by the A1 antenna and, after detection, enters the input of the EM sensor. The EM sensor contains a preamplifier and a comparator, and the signal received at the output of the sensor takes the shape as shown in black in the graph.

After the comparator, the signal goes to the ADC, there digitized for the MCU. MCU processes PD signals in amplitude and time from the beginning of the phase interval received from the phase sensor.

There are various methods of application and placement of receiving devices for registering PD: configuration of 4 receiving devices Y-shaped and quadratic in work [1], installation of an antenna array and data collection systems on a mobile platform [4-5], simultaneous use of devices with acoustic sensors in transformers [2], etc.

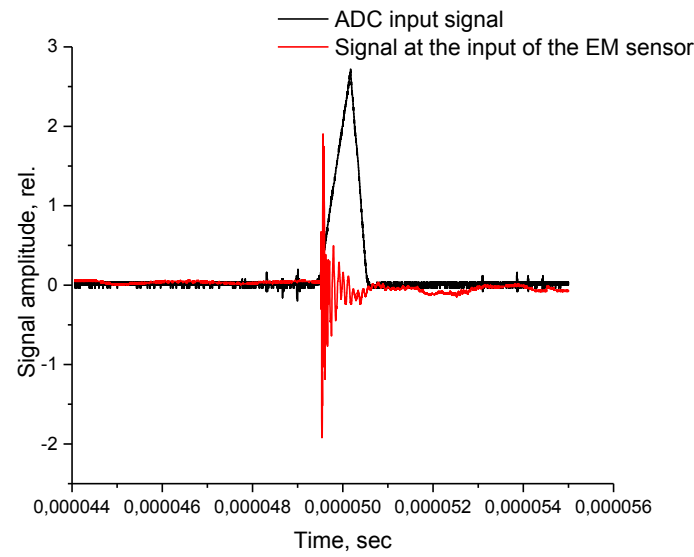

Fig. 2. Signal before and after NDD processing.

In our case, a complex of three NDDs, one device each on a phase wire, is a receiving antenna array (Fig. 3). The time difference of arrival (TDOA) between the received signals at each of the corresponding NDDs is determined, which allows the three-dimensional position of the electrical discharge source to be inferred by processing the TDOA values. This method is used in this work. Noncontact diagnostic devices are installed on each phase wire $\left(D_{1}-D_{3}\right)$ at a certain known distance from the highvoltage insulator $-r_{i}$, where $i$ is the serial number of the sensor $(i=1-3)$. The sensor works on power lines from 10 $\mathrm{kV}$.

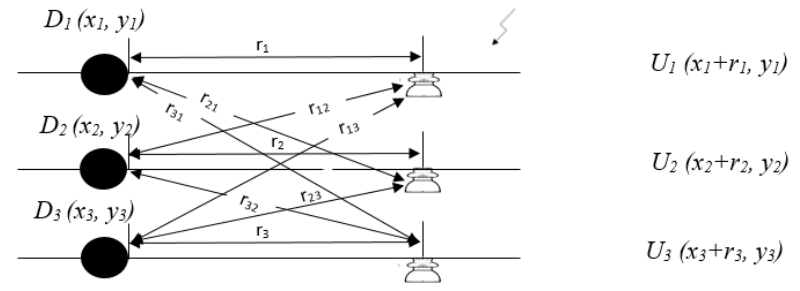

Fig. 3. NDD installation diagram.

The time difference localization method consists of two main stages, namely, the estimation of the difference in arrival time and the solution of nonlinear equations of the time difference $[1,5,13,14]$. This method is often used for local positioning of mobile devices. The method is based on measuring the difference in transmission time of radiation from $\mathrm{PD}$ to NDD, with time synchronization and a predetermined location. Knowing the difference in signal acquisition time, the distance from the PD source to the NDD is calculated using mathematical processing.

There are various computational algorithms that can be used to locate partial discharges in three dimensions: Newton-Raphson method [15], spatial grid search algorithm [13-14], Chan direct solution method [12], least squares method [1], Bancroft method [1].

\section{Defective insulator determination method}

The paper proposes an application of the well-proven standard least squares method. To simplify calculations, we take the high-voltage insulator as a material point, i.e. any point of the insulator is equal to the point of its suspension.

Knowing the coordinates of each NDD installed on the phase conductor (Fig. 2), it is possible to calculate the propagation time from the source of partial discharges to the corresponding NDDs using the basic formula $D=v$. $t$, where $D$ is the distance, $v$ is the propagation velocity, and $t$ is the time distribution. This method is described by the formula (1):

$$
\left(x-x_{i}\right)^{2}+\left(y-y_{i}\right)^{2}+\left(z-z_{i}\right)^{2}=\left(V_{e}^{*} t_{i}\right)^{2}
$$

where $\left(x_{i}, y_{i}, z_{i}\right)$ are the coordinates of the $i$-th diagnostic device in Cartesian space, $(x, y, z)$ are the true coordinates of the partial discharge event, ve is the speed of the electromagnetic wave, ti is the "time of flight" of the propagating PD signal from its source to the $i$-th sensor. Since measurements are made in 2 dimensions, the $\mathrm{z}$ 
coordinate is not taken into account and formula (1) will take the following form:

$$
\left(x-x_{i}\right)^{2}+\left(y-y_{i}\right)^{2}=\left(V_{e} * t_{i}\right)^{2}
$$

Let the time of flight from the PD source to NDD $D_{1}$ be equal to $T$, and the difference in arrival time between devices D1, D2, D3 is equal to $\tau 1 \mathrm{n}$. For our case, for three NDDs, a system of equations is obtained [16]:

$$
\begin{aligned}
& \left(x-x_{1}\right)^{2}+\left(y-y_{1}\right)^{2}=\left(V_{e}^{*} T\right)^{2} \\
& \left(x-x_{2}\right)^{2}+\left(y-y_{2}\right)^{2}=\left(V_{e}^{*}\left(T+\tau_{12}\right)\right)^{2} \\
& \left(x-x_{3}\right)^{2}+\left(y-y_{3}\right)^{2}=\left(V_{e}^{*}\left(T+\tau_{13}\right)\right)^{2}
\end{aligned}
$$

The position of the PD source $(x, y)$ can be calculated using the least squares method:

$$
S(X)=\sum_{i=1}^{N}\left(Y_{i}(X)\right)^{2}
$$

In the least squares method, the standard definition of $\mathrm{Yi}(\mathrm{X})$ is expressed as

$$
Y_{i}(X)=\sqrt{\left(x-x_{i}\right)^{2}+\left(y-y_{i}\right)^{2}}-\left(V_{e}^{*}\left(T+\tau_{1 i}\right)\right)
$$

The least squares method is iterative and gives an approximate solution to the equations based on the number of iterations and starting from an initial value, which is improved at each iteration using the error boundary until a convergent solution is found or until the maximum number of iterations is reached.

After determining the number of PDs, their intensity and location, the obtained distribution is compared with that for serviceable, pre-defective and defective insulators. Using the developed technique, the rate of the aging process and the development of various defects is determined, which makes it possible to predict the residual life of the high-voltage insulator.

\section{Conclusion}

Diagnosing the presence of partial discharges in insulation and determining their location using contactless diagnostic devices installed on phase wires will allow detecting defects in the early stages of their appearance, determining which high-voltage insulator has a defect, and predicting the residual life of a high-voltage insulator. Electric power companies will be able to receive this information remotely and plan in advance for repair or replacement of equipment, which will significantly increase economic efficiency.

At the moment, there is a prototype NDD, which has been tested in laboratory conditions, it is planned to develop models for assessing the residual life of highvoltage insulators and conduct field tests.
The reported study was funded by RFBR according to the research project № 20-38-90145.

\section{References}

1. O. E. Mountassir, B. G. Stewart, A. J. Reidc, S. G. McMeekind. Electric Power Systems Research, 143, pp. 110-120 (2017)

2. H. Chai, Sh. Lu, B.T. Phung, S. Mitchell. Comparative study of partial discharge localization based on uhf detection methods // 25th International Conference on Electricity Distribution, 2019, Madrid, pp. 1076-1080. 3. M. D. Judd, L. Yang, Ian B. B. Hunter. IEEE Electrical Insulation Magazine. 21, 2 (2005).

4. P. Li, W. Zhou, Sh. Yang, Y. Liu, Ya. Tian, Yo. Wang. IET Sci. Meas. Technol., 11, 3 (2017).

5. Ming-Xiao Zhu, Yan-Bo Wang, Qing Liu, Jia-Ning Zhang, Jun-Bo Deng, Guan-Jun Zhang. IEEE Transactions on Dielectrics and Electrical Insulation, 24, 1 (2017).

6. A.V. Golenischev-Kutuzov, L.V. Akhmetvaleeva G.R. Enikeeva, D.A. Ivanov, A.V. Semennikov, G.D. Mardanov, Power engineering: research, equipment, technology. 22, 2 (2020).

7. A.V. Golenischev-Kutuzov, D.A. Ivanov, A.A. Potapov, V.I. Krotov, Power engineering: research, equipment, technology, 21, 4 (2019).

8. J.P. Steiner, P.H. Reynolds, W.L. Weeks. IEEE Transactions on Dielectrics and Electrical Insulation. 27, pp. 44-59 (1992).

9. Y. Tian, P. Lewin, A. Davies, S. Sutton, S. Swingler. IEEE Trans. Dielectr. Electr. Insul. 10, pp. 343353, (2003).

10. R. Mardiana, C.Q. Su. IEEE Trans. Dielectr. Electr. Insul., 17, pp. 1738-1746, (2010).

11. P. Wagenaars, P.A.A.F. Wouters, P.C.J.M. Van der Wielen, F. Steennis. IEEE Trans. Power Deliv., 26, pp. 1064-1071, (2011).

12. H.H. Sinaga, B.T. Phung, T.R. Blackburn. IEEE Trans. Dielectr. Electr. Insul., 2012, 19, pp. 1891-1900, (2012).

13. H. J. Hou, G. H. Sheng, X. C. Jiang. IEEE Trans. Power Del., 28, 3 (2013)

14. S. Zheng, C. Li, Z. Tang, et al. IEEE Trans. Dielectr. Electr. Insul., 21, 1 (2014).

15. I. E. Portugues, P. J. Moore, I. A. Glover. IEEE Trans. Power Del., 24, 1 (2009).

16. Y. Lu, X. Tan, X. Hu. IEE Proceedings - Science, Measurement and Technology, 147, 2 (2000). 